\title{
Biochemical characterization of novel glucokinase mutations isolated from Spanish maturity-onset diabetes of the young (MODY2) patients
}

\author{
Itziar Estalella $\cdot$ Maria Adelaida Garcia-Gimeno • \\ Alberto Marina $\cdot$ Luis Castaño $\cdot$ Pascual Sanz
}

Received: 3 January 2008/Accepted: 14 February 2008/Published online: 6 March 2008

(C) The Japan Society of Human Genetics and Springer 2008

\begin{abstract}
Mature-onset diabetes of the young, type 2 (MODY2) is associated with mutations in the glucokinase (GCK) gene that result in impaired glucokinase activity. Although more than 200 inactivating GCK mutations have been reported, only less than $20 \%$ of these mutations have been functionally characterized. In this work, we describe the biochemical characterization of six missense glucokinase mutations associated with MODY2 from Spanish patients, namely, Y61S, V182L, C233R, E265K, A379V, and K420E. All these mutations produced enzymes that presented reduced enzymatic activity in various degrees, from a mild affectation (K420E) to a more severe effect (C233R). Mutation severity correlated with the importance of the structural changes introduced by the mutations. For example, C233R affected a critical residue of the active center of the enzyme and rendered a protein with undetectable enzymatic activity. These data add new information on the structure-function relationship of human glucokinase.
\end{abstract}

Keywords MODY2 - Glucokinase · Inactivating mutation $\cdot$ Enzyme kinetics

I. Estalella and M.A. Garcia-Gimeno contributed equally to this work.

I. Estalella · L. Castaño

Endocrinology and Diabetes Research Group,

Hospital de Cruces and University of Basque Country,

Bizkaia, Basque Country, Spain

M. A. Garcia-Gimeno · A. Marina · P. Sanz $(\bowtie)$

Instituto de Biomedicina de Valencia (CSIC)

and Centro de Investigación Biomédica en Red de Enfermedades

Raras (CIBERER), Jaime Roig 11, 46010 Valencia, Spain

e-mail: sanz@ibv.csic.es

\section{Introduction}

Glucokinase B (GlkB, hexokinase IV) is the first enzyme of the glycolytic pathway in pancreatic $\beta$-cells. It phosphorylates the glucose that enters these cells through the GLUT2 glucose transporter to produce Glu-6P, which is then used to obtain the energy necessary for cell viability. The kinetic properties of GLUT2 (high-capacity, lowaffinity glucose transporter) and GlkB (low affinity for glucose and absence of allosteric regulation by Glu-6P) define GlkB as the key regulator of glucose consumption. For this reason, several authors have considered GlkB as the "glucose sensor" of pancreatic $\beta$-cells (Matschinsky et al. 1993, 1996, 1998, 2002; Zelent et al. 2005). In addition, a positive correlation between the functionality of GlkB and the ability of pancreatic $\beta$-cells to respond to increased levels of glucose in blood by enhancing insulin production and secretion has been described. In this way, GlkB participates in maintaining blood glucose concentration around $5 \mathrm{mM}$. Mutations in the glucokinase (GCK) gene that increase the affinity of the enzyme for glucose decrease the threshold for glucose-stimulated insulin secretion (GSIS) and therefore trigger insulin secretion at lower concentrations of glucose in blood. These mutations are associated with both mild and severe forms of persistent hyperinsulinemic hypoglycemia in infancy (PHHI$\mathrm{GK})$. On the other hand, inactivating mutations in one or both alleles of the GCK gene leads to an enzyme with low activity, which increases the threshold for GSIS from its physiological setting of $5 \mathrm{mM}$. These types of mutations are associated with a mild form of diabetes mellitus in young people known as maturity-onset diabetes of the young, type 2 (MODY2) or with severe permanent neonatal diabetes mellitus (PNDM) (see Matschinsky 2002; Zelent et al. 2005; Gloyn 2003, for review). 
MODY2 is a form of diabetes mellitus characterized by an onset that usually appears before 25 years of age and abnormal $\beta$-cell function and insulin secretion (Velho et al. 2004). The MODY2 phenotype is characterized by a mild, nonprogressive form of hyperglycemia present from birth, often asymptomatic and only detected later in life. MODY2 patients are usually treated with diet alone and rarely develop diabetic-associated complications (Velho et al. 2004). Nearly 200 mutations have been already identified as inactivating GCK mutations. They are almost evenly distributed along the ten exons that comprise the GCK gene and include missense, nonsense, and frameshift mutations produced by deletions or insertions. However, only less than $20 \%$ of these mutations have been functionally characterized (Gloyn 2003; Velho et al. 2004; Gloyn et al. 2004). Despite considerable differences in the effect of the mutations on the in vitro activity of the enzyme, it is generally accepted that the MODY2 phenotype is quite homogeneous (Miller et al. 1999; Stride et al. 2002).

The crystal structure of human GlkB has been recently characterized (Kamata et al. 2004). These results indicate that the protein has a large and a small domain separated by a deep cleft. Glucose binds to the interdomain cleft, composed of residues of the large domain (Glu256 and Glu290), the small domain (Thr168 and Lys169), and the connecting region I (Asn204 and Asp205). Upon binding to substrates [glucose and adenosine triphosphate (ATP)], GlkB undergoes a conformational change that brings the large and small domains physically closer, resulting in a closed, active conformation. This structural model has helped in the understanding of the abnormal biochemistry of different GCK missense mutations (Pedelini et al. 2005; Galan et al. 2006).

In this study, we report the biochemical characterization of six GCK missense mutations found in Spanish MODY2 families (Estalella et al. 2007) and evaluate the impact of the mutation at the structural level by introducing these modifications into the closed active and superopen inactive GlkB structure models described recently (Kamata et al. 2004).

\section{Materials and methods}

Subjects

A total of 21 subjects from nine families (Estalella et al. 2007) with one of the following six GCK mutations (Y61S, V182L, C233R, E265K, A379V, and K420E) were included in this study. These mutations were selected among the different mutations identified in the previous study (Estalella et al. 2007) because they were novel missense mutations (except E265K) that were representative of different areas of the molecular structure of GCK. All patients met the classic MODY2 diagnostic criteria, presenting a defect in glucose homeostasis, absence of autoimmunity markers, and an autosomal dominant mode of inheritance (Velho and Froguel 1997). The human ethic committee of Cruces hospital approved the study, and all analyses were performed after informed consent was obtained from individuals or their parents.

Site-directed mutagenesis

All the mutants used in this study were obtained as described (Pedelini et al. 2005) using the QuickChange site-directed mutagenesis kit from Stratagene (La Jolla, CA, USA) and the oligonucleotides described in Table 1.

Purification of GST-fusion proteins and kinetic determinations

Purification of the mutated glutathione S-transferase (GST)-GlkB fusion proteins was carried out as described
Table 1 Oligonucleotides used in this study

The modified codon is underlined

\begin{tabular}{|c|c|}
\hline Name & Sequence \\
\hline Y61S-1 & 5'-CAGTGTGAAGATGCTGCCCACCTCCGTGCGCTCCACCCCAGAAGG-3' \\
\hline Y61S-2 & 5'-CCTTCTGGGGTGGAGCGCACGGAGGTGGGCAGCATCTTCACACTG-3' \\
\hline V182L-1 & 5'-GGAGCAGAAGGGAACAATGTCTTGGGGCTTCTGCGAGACGCTATC-3' \\
\hline V182L-2 & 5'-GATAGCGTCTCGCAGAAGCCCCAAGACATTGTTCCCTTCTGCTCC-3' \\
\hline C233R-1 & 5'-GGCACGGGCTGCAATGCCCGCTACATGGAGGAGATGCAGAATGTG-3' \\
\hline C233R-2 & 5'-CACATTCTGCATCTCCTCCATGTAGCGGGCATTGCAGCCCGTGCC-3' \\
\hline E265K-1 & 5'-GCCTTCGGGGACTCCGGCAAGCTGGACGAGTTCCTGCTGGAGTATG-3' \\
\hline E265K-2 & 5'-CATACTCCAGCAGGAACTCGTCCAGCTTGCCGGAGTCCCCGAAGGC-3' \\
\hline A379V-1 & 5'-GAGAGCGTGTCTACGCGCGCTGTGCACATGTGCTCGGCGGGGCTGG-3' \\
\hline A379V-2 & 5'-CCAGCCCCGCCGAGCACATGTGCACAGCGCGCGTAGACACGCTCTC-3' \\
\hline K420E-1 & 5'-GTACAAGCTGCACCCCAGCTTCGAGGAGCGGTTCCATGCCAGCGTG-3' \\
\hline K420E-2 & 5'-CACGCTGGCATGGAACCGCTCCTCGAAGCTGGGGTGCAGCTTGTAC-3' \\
\hline
\end{tabular}


(Pedelini et al. 2005). The kinetic parameters of the recombinant GST-GlkB proteins were also measured as described (Pedelini et al. 2005).

Prediction of structural effects of glucokinase mutations

Crystal coordinates from the closed active (1V4S) and superopen inactive (1V4T) conformation of GlkB (Kamata et al. 2004) were visualized and mutations evaluated using O program (Jones and Kjeldgaard 1997). Figure 2 was generated with MolScript (Kraulis 1991) and Render 3D (Merritt and Bacon 1997) programs using the original or Oprogram-modified PDBs.

\section{Results and discussion}

Kinetic parameters of novel GCK missense mutations

Selected missense mutations from Spanish MODY2 families (Y61S, V182L, C233R, E265K, A379V, and K420E; see Table 2) were introduced in GCK complementary DNA (cDNA) by site-directed mutagenesis using appropriated oligonucleotides. Mutated cDNAs were subcloned into plasmid pGEX6P-1 to produce GST-GlkB proteins that were purified from bacteria. We then compared the kinetic parameters of the mutated forms with those of the wild-type GCK (Table 2, Fig. 1a). Since in regular conditions of glucose homeostasis the concentration of glucose in human blood is around $5 \mathrm{mM}$ and there are saturating concentrations of ATP, we assumed that the activity of the enzyme at $5 \mathrm{mM}$ glucose and saturating concentrations of ATP $(5 \mathrm{mM})$ is a good parameter to correlate enzyme activity with its performance under physiological conditions (Pedelini et al. 2005) (Fig. 1b). All six missense mutations presented reduced enzymatic activity in various degrees, from a mild affectation to a more severe effect.
The K420E mutation was the mildest; it had a lower glucose affinity (S0.5: $11.6 \mathrm{mM}$ ) in comparison with the wild type (S0.5: $7.6 \mathrm{mM}$ ). Interestingly, activity of the mutated form at $5 \mathrm{mM}$ glucose was almost half that of the wild type, suggesting a poor performance as a glucose-phosphorylating enzyme under physiological conditions (Fig. 1b).

The E265K and A379V mutations had affected both $V_{\max }(37.9$ and $28.7 \mathrm{U} / \mathrm{mg}$, respectively) and $\mathrm{S} 0.5$ (11.5 and $13.7 \mathrm{mM}$, respectively). In addition, A379V had a lower ATP affinity (Km $0.69 \mathrm{mM})$. The activity of the E265K mutated form at $5 \mathrm{mM}$ glucose was also half that of the wild type, whereas the activity of the A379V mutated form was even poorer (Fig. 1b). Mutations V182L and Y61S severely affected glucose affinity (S0.5: 28.8 and $39.2 \mathrm{mM}$, respectively) and the $V_{\max }$ of the mutated forms (16.7 and 7.2 U/mg, respectively). Both mutations increased ATP affinity (KmATP: 0.16 and $0.21 \mathrm{mM}$, respectively). Activity of the mutated forms at $5 \mathrm{mM}$ glucose was very poor (Fig. 1b), suggesting that these enzymes had a very poor performance as glucose-phosphorylating enzymes under physiological conditions. Finally, when we assayed the C233R mutant activity, we were unable to detect any activity, suggesting that this mutation had abolished the glucose-phosphorylating activity of the enzyme.

\section{Prediction of structural effects of GCK mutations}

To understand the changes in the kinetic parameters of the mutated enzymes, we evaluated the impact of the mutation at the structural level by introducing these modifications into the closed active and superopen inactive GlkB structure models described recently (Kamata et al. 2004). Figure 2 shows the effects of the mutations on the closed active conformation of the enzyme.

The K420 residue (Fig. 2f) is located in the $\alpha 12$ helix of the large domain and is exposed to the solvent in both the

Table 2 Kinetic parameters of mutated forms of glucokinase

\begin{tabular}{lclcccc}
\hline Nucleotide change & Exon & Amino acid change & $V_{\max }(\mathrm{U} / \mathrm{mg})$ & S0.5 $(\mathrm{mM})$ & Hill number & KmATP $(\mathrm{mM})$ \\
\hline & & Wild type & $44.1 \pm 2.0$ & $7.6 \pm 0.3$ & $1.42 \pm 0.04$ & $0.45 \pm 0.03$ \\
TAC $>$ TCC & 2 & Y61S & $7.2 \pm 0.6$ & $39.2 \pm 0.3$ & $1.50 \pm 0.17$ & $0.21 \pm 0.01$ \\
GTG $>$ TTG & 5 & V182L & $16.7 \pm 0.1$ & $28.8 \pm 2.7$ & $1.61 \pm 0.05$ & $0.16 \pm 0.03$ \\
TGC $>$ CGC & 7 & C233R & ND & ND & ND & ND \\
GAG $>$ AAG & 7 & E265K & $37.9 \pm 3.2$ & $11.5 \pm 0.5$ & $1.37 \pm 0.07$ & $0.40 \pm 0.07$ \\
GCG $>$ GTG & 9 & A379V & $28.7 \pm 7.5$ & $13.7 \pm 0.1$ & $1.45 \pm 0.02$ & $0.69 \pm 0.13$ \\
AAG $>$ GAG & 10 & K420E & $41.3 \pm 5.7$ & $11.6 \pm 0.3$ & $1.47 \pm 0.04$ & $0.41 \pm 0.05$ \\
\hline
\end{tabular}

Glucose-dependent activity $\left(V_{\max }, \mathrm{S} 0.5\right.$, Hill number) and adenosine triphospate (ATP)-dependent activity (Km) parameters were determined in purified glutathione S-transferase glucokinase B (GST-GlkB) fusion proteins. Values are the mean of at least two different kinetic determinations for each purification procedure for each mutant \pm standard deviation. The nucleotide change and the exon location for each mutant are also indicated

$N D$ not detectable 

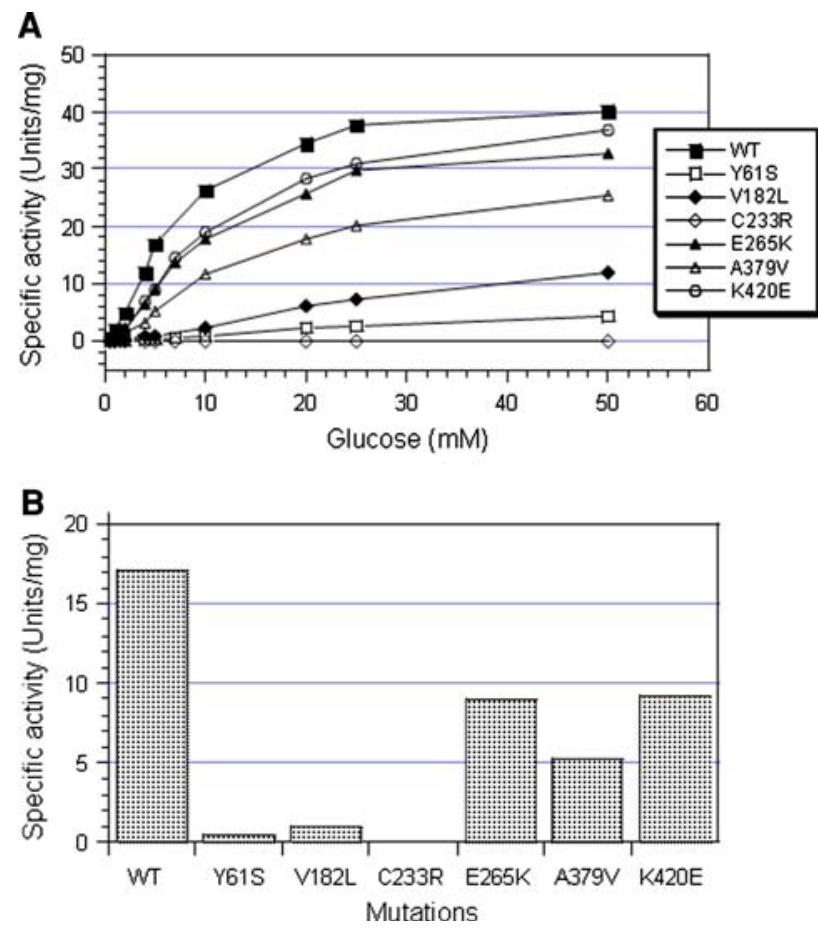

Fig. 1 Enzymatic functionality of the mutated forms of glucokinase (GCK). a Glucose-dependent activity of mutated forms of GCK. b Specific activity of mutated forms of glucokinase at $5 \mathrm{mM}$ glucose and saturating concentrations of adenosine triphosphate (ATP) $(5 \mathrm{mM})$ at $30^{\circ} \mathrm{C}$. A representative experiment of at least two different kinetic determinations for each purification procedure is shown in each case

closed and the superopen conformations. In the K420E mutation, there is a change in the charge of the side chain (from the positive Lys to the negative Glu residue), and perhaps this may affect its interaction with other negatively charged residues in the surrounding residues, such as E421 and E440. The E265 residue (Fig. 2d) is also located in the large domain and is exposed to the solvent. The E265K mutation would affect putative interactions with residues of the $\alpha 2$ helix, such as E46 and R40. Recently, a closely related mutation, G264S (Massa et al. 2001), has been characterized, which also has minor effects on the kinetics of the enzyme (S0.5: $9.76 \mathrm{mM}$; KmATP: $0.48 \mathrm{mM}$ ) (Gloyn et al. 2004). Since at the structural level the modifications introduced by all these three mutations (K420E, E265K and G264S) are minor, perhaps this is the reason the kinetic properties of the mutated enzymes are not greatly affected. Recently, characterization of the E265K mutation was described. The authors suggested that although the impact of the mutation was minor at the level of the kinetics of the enzyme, it strongly affected protein stability, suggesting a possible structural level defect of this mutant protein (Galan et al. 2006).

The A379 residue (Fig. 2e) is located in the $\alpha 11$ helix of the large domain at the back of the ATP binding site. Since
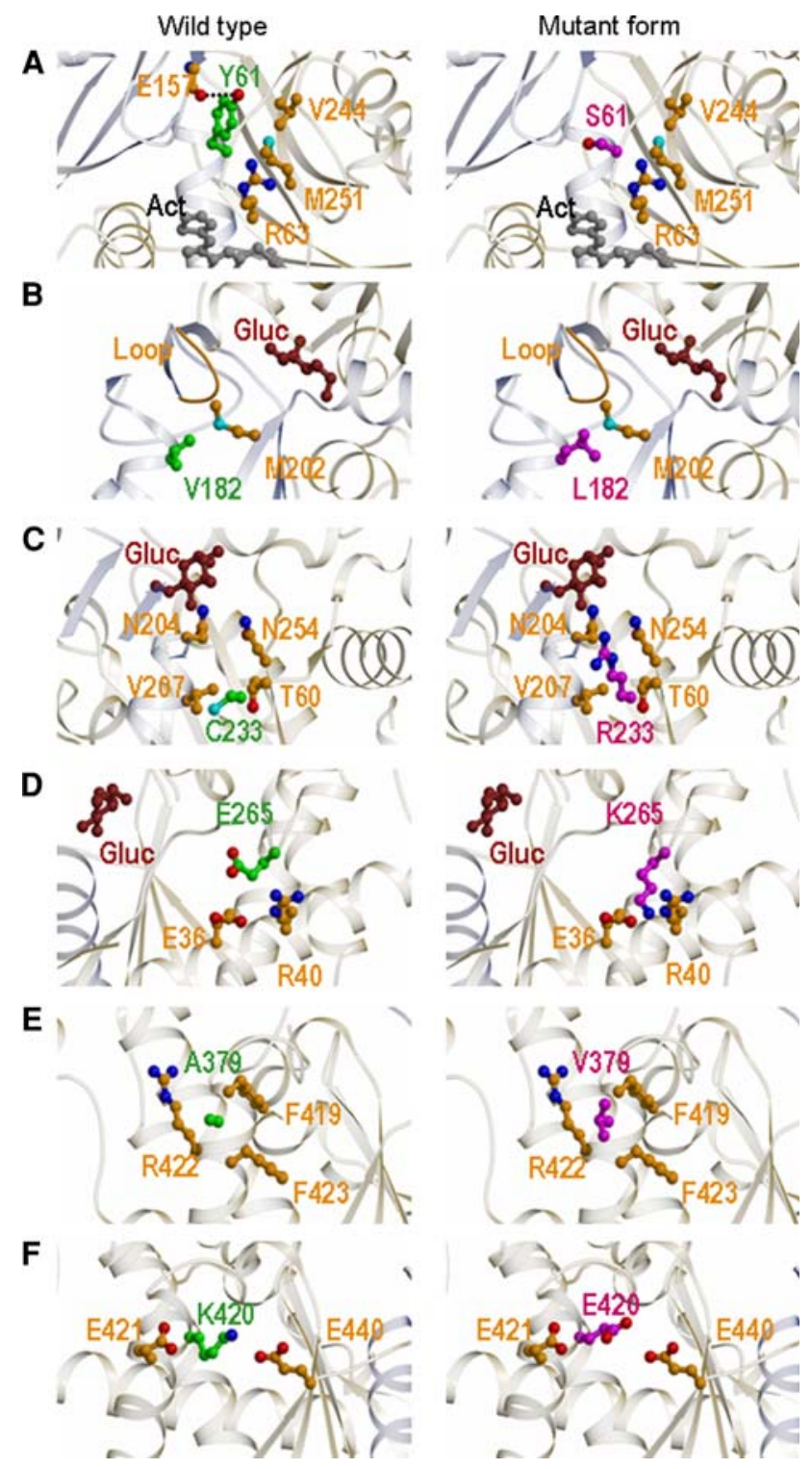

Fig. 2 Effects of the mutations on the structure model of glucokinase. a-f The different mutations were studied at the structural level by introducing these modifications into the closed active glucokinase B (GlkB) structure model. Wild-type residues are green, whereas the mutated residues are magenta. Interacting residues are orange. In some panels, the position of the glucose $(G l u c)$ or the glucokinase activator $(A c t)$ molecules is also indicated

this residue is very close to residues of the $\alpha 12$ helix (such as F419, R422, and F423), the increase in size of the side chain of the new valine residue in the A379V mutation would force a reorganization of this protein region. For this reason, the kinetic properties of the mutated enzyme should be more affected. It is worth noting that a closely related mutation, A378V, has been recently described as a GCK mutation, found in a case of permanent neonatal diabetes (Njolstad et al. 2003). The A378V is buried in the large domain hydrophobic core and directly oriented toward the 
ATP binding site, and shows a severe defect in enzyme kinetics (S0.5: $584 \mathrm{mM}$; KmATP: $8.08 \mathrm{mM}$ ) (Gloyn et al. 2004). Both modest Ala to Val mutations reflect the importance of the correct disposition of the $\alpha 11$ helix, although the relative orientation of the residue has a different impact on the structure of the protein and consequently on the kinetics of the enzyme.

The V182 residue (Fig. 2b) is located in the $\alpha 4$ helix base of the small domain, following a catalytic loop that is involved in glucose binding site formation. In the superopen conformation, the glucose molecule is absent, and consequently, this loop is disorganized. The transition between superopen and closed conformations involves a $\sim 90^{\circ}$ displacement of the $\alpha 4$ helix, pivoting over its base (where V182 localizes), in order to accommodate the catalytic loop. The V182L mutation introduces a residue with bigger side chain and higher hydrophobicity that might disturb an area packed with the side chains of residues D160 and K161 (in the loop) and M202. In this way, it would destabilize the catalytic loop disposition in the closed conformation of the enzyme. Furthermore, the change V182L might interfere in the transition between the different catalytic conformations. For this reason, the mutated protein should have lower $V_{\text {imax }}$ and glucose affinity and higher Hill number than the wild type. The substitution of Val by a Met residue (V182M) has also been reported (Costa et al. 2000). In this case, the mutated enzyme also displays lower $V_{\max }$ and lower glucose affinity (S0.5: $83.9 \mathrm{mM}$ ) than the wild type (Gloyn et al. 2004). On the contrary, both V182L and V182M mutations displayed a higher ATP affinity (KmATP: 0.16 and $0.10 \mathrm{mM}$, respectively).

The Y61 residue (Fig. 2a) is located in the connexion between the small and large domains. In the closed conformation, the bulky hydrophobic Tyr side chain may make hydrophobic interactions with surrounding residues, such as R63, V244, and M251, and a hydrogen bridge bond with E157 main-chain oxygen, stabilizing the closed conformation of the enzyme. In the Y61S mutation, the big hydrophobic Tyr residue is changed to a small polar Ser residue that no longer maintains these interactions, destabilizing the closed conformation of the enzyme. This would result in a decrease in the $V_{\max }$ and the glucose affinity of the mutated enzyme. In a closely related mutation, V62A (Njolstad et al. 1998), the kinetic parameters were similarly affected (S0.5: $27.4 \mathrm{mM}$; KmATP: $0.20 \mathrm{mM}$ ) (Gloyn et al. 2004), suggesting that this area may play an important role in maintaining the closed conformation of the enzyme. Interestingly, a V62M mutation shows higher glucose affinity (S0.5: $4.88 \mathrm{mM}$ ), although the mutation was isolated from patients with hyperglycemia (Gloyn et al. 2005).

Finally, the C233 residue (Fig. 2c) is located in the $\beta 10$ sheet that forms part of the hydrophobic interface between small and large domains in the base of the active center of the enzyme (glucose binding site). In the C233R mutation,
Table 3 Clinical

characteristics of subjects with the different mature-onset diabetes of the young, type 2 (MODY2) mutations ordered by decreasing in vitro enzymatic activity

IGT impaired glucose tolerance, $O H A$ oral hypoglycemic agents, $F P G$ fasting plasma glucose, 2- $h$ OGTT plasma glucose $2 \mathrm{~h}$ after an oral glucose tolerance test

\begin{tabular}{|c|c|c|c|c|c|c|c|}
\hline Mutation & Age & $\begin{array}{l}\text { Years since } \\
\text { diagnosis }\end{array}$ & FPG (mmol/l) & $\begin{array}{l}\text { 2-h OGTT } \\
(\mathrm{mmol} / \mathrm{l})\end{array}$ & $\mathrm{HbA}_{1 \mathrm{c}}(\%)$ & Diagnosis & $\begin{array}{l}\text { Actual } \\
\text { treatment }\end{array}$ \\
\hline K420E & 10 & 5 & 6.4 & 10.2 & 6.4 & IGT & Diet \\
\hline K420E & 14 & 2 & 6.7 & 10.1 & 6.0 & IGT & Diet \\
\hline K420E & 68 & - & 6.8 & 10.5 & - & IGT & Diet \\
\hline K420E & 44 & 29 & 6.0 & 10.9 & - & IGT & Diet \\
\hline E265K & 25 & 17 & 7.2 & - & 7.2 & Diabetes & Diet \\
\hline E265K & 36 & 21 & 6.6 & 8.1 & & IGT & Diet \\
\hline E265K & 39 & 20 & 7.8 & - & 6.5 & Diabetes & Diet \\
\hline E265K & 42 & 11 & 7.8 & - & 6.6 & Diabetes & Diet \\
\hline A379V & 39 & 9 & 7.4 & 12.1 & - & Diabetes & Diet \\
\hline A379V & 39 & 16 & 5.7 & 12.5 & 5.7 & Diabetes & Diet \\
\hline A379V & 68 & 12 & 7.6 & - & - & Diabetes & Diet \\
\hline V182L & 10 & 7 & 7.5 & - & 5.6 & Diabetes & Diet \\
\hline V182L & 11 & 3 & 7.3 & - & & Diabetes & Diet \\
\hline V182L & 21 & 11 & 7.1 & 9.5 & 6.8 & Diabetes & Diet \\
\hline V182L & 49 & 31 & 7.2 & - & 6,2 & Diabetes & OHA \\
\hline Y61S & 27 & 23 & 7.5 & 11.4 & 5.7 & Diabetes & Diet \\
\hline Y61S & 45 & 10 & 7.8 & - & 5.8 & Diabetes & Diet \\
\hline C233R & 28 & 17 & 5.6 & 9.3 & - & IGT & Diet \\
\hline C233R & 30 & 17 & 6.2 & 10.9 & - & IGT & Diet \\
\hline C233R & 54 & 30 & - & - & 5.1 & Diabetes & OHA \\
\hline C233R & 62 & 17 & - & - & - & Diabetes & Insulin \\
\hline
\end{tabular}


the new Arg residue has a huge side chain in comparison with the small original Cys. To accommodate the new side chain, an important reorganization in the surrounding area must occur, destabilizing the active centre and consequently affecting severely the activity of the enzyme.

\section{Phenotype/genotype relationship}

Clinical characteristics of patients carrying the corresponding GCK mutations are shown in Table 3. To determine whether the degree of biochemical in vitro defect in GCK activity was related to phenotype severity, mutations were ordered according to the magnitude of the change in enzymatic activity. However, no differences were observed among patients regarding clinical characteristics. Average fasting plasma glucose (FPG) $(6.95 \pm 0.8 \mathrm{mmol} /$ 1) was found in all cases and was similar to previously reported values (Stride et al. 2002). Although most MODY2 patients required only diet for control, three were treated with oral hypoglycaemic agents (OHA) or insulin, and these patients carried severe changes in the in vitro GCK activity. However, as there were other patients carrying the same mutation who only required diet for control, we cannot establish a clear relationship between the severity of the biochemical defect and the actual diagnosis and treatment.

In summary, our data confirmed that MODY2-associated GCK mutations can be due to modifications in conserved residues of the active site, as in the case of C233R; to modifications that distort the structure of the protein, as in the case of $\mathrm{A} 379 \mathrm{~V}, \mathrm{~V} 182 \mathrm{~L}$, and Y61S; or to modifications in residues of the periphery of the molecule that may disrupt interactions with other residues of the protein or with other interacting proteins, as in the case of $\mathrm{K} 420 \mathrm{E}$ and E265K, as previously suggested (Miller et al. 1999; Velho and Froguel 1997). These data add new information on the structure-function relationship of human glucokinase.

Akcnowledgments We thank the Spanish MODY Group: Pedro Martul (Hospital Cruces-Bizkaia), María José López (Hospital Clinico-Valencia), Cristina Luzuriaga (Hospital Valdecilla-Santander), Rafael Ruiz Cano (Hospital Universitario-Albacete) and Federico Vázquez (Hospital Cruces-Bizkaia) for their contribution. This work was supported by grants from the Instituto de Salud Carlos III (PIO50423) and Spanish Ministry of Education and Science (SAF2005-00852) to PS and by grant RD06/0015/0031 from the Instituto de Salud Carlos III to LC.

\section{References}

Costa A, Bescos M, Velho G, Chevre J, Vidal J, Sesmilo G, BellanneChantelot C, Froguel P, Casamitjana R, Rivera-Fillat F et al (2000) Genetic and clinical characterisation of maturity-onset diabetes of the young in Spanish families. Eur J Endocrinol 142:380-386
Estalella I, Rica I, de Nanclares GP, Bilbao JR, Vazquez JA, San Pedro JI, Busturia MA, Castano L (2007) Mutations in GCK and HNF-1alpha explain the majority of cases with clinical diagnosis of MODY in Spain. Clin Endocrinol 67:538-546

Galan M, Vincent O, Roncero I, Azriel S, Boix-Pallares P, DelgadoAlvarez E, Diaz-Cadorniga F, Blazquez E, Navas MA (2006) Effects of novel maturity-onset diabetes of the young (MODY)associated mutations on glucokinase activity and protein stability. Biochem J 393:389-396

Gloyn AL (2003) Glucokinase (GCK) mutations in hyper- and hypoglycemia: maturity-onset diabetes of the young, permanent neonatal diabetes, and hyperinsulinemia of infancy. Hum Mutat 22:353-362

Gloyn AL, Odili S, Buettger C, Njolstad PR, Shiota C, Magnuson MA, Matschinsky FM (2004) Glucokinase and the regulation of blood sugar. In: Matschinsky FM, Magnuson MA (eds) Glucokinase and glycemic disease. From basics to novel therapeutics, Karger, Basel, pp 92-109

Gloyn AL, Odili S, Zelent D, Buettger C, Castleden HA, Steele AM, Stride A, Shiota C, Magnuson MA, Lorini R et al (2005) Insights into the structure and regulation of glucokinase from a novel mutation (V62M), which causes maturity-onset diabetes of the young. J Biol Chem 280:14105-14113

Jones TA, Kjeldgaard MO (1997) Electron-density map interpretation. Methods Enzymol 277:173-208

Kamata K, Mitsuya M, Nishimura T, Eiki J, Nagata Y (2004) Structural basis for allosteric regulation of the monomeric allosteric enzyme human glucokinase. Structure 12:429-438

Kraulis PJ (1991) MOLSCRIPT: a program to produce both detailed and schematic plots of protein structures. J Appl Crystallogr 24:946-950

Massa O, Meschi F, Cuesta-Munoz A, Caumo A, Cerutti F, Toni S, Cherubini V, Guazzarotti L, Sulli N, Matschinsky FM et al (2001) High prevalence of glucokinase mutations in Italian children with MODY. Influence on glucose tolerance, first-phase insulin response, insulin sensitivity and BMI. Diabetes Study Group of the Italian Society of Paediatric Endocrinology and Diabetes (SIEDP). Diabetologia 44:898-905

Matschinsky F, Liang Y, Kesavan P, Wang L, Froguel P, Velho G, Cohen D, Permutt MA, Tanizawa Y, Jetton TL et al (1993) Glucokinase as pancreatic beta cell glucose sensor and diabetes gene. J Clin Invest 92:2092-2098

Matschinsky FM (1996) Banting Lecture 1995. A lesson in metabolic regulation inspired by the glucokinase glucose sensor paradigm. Diabetes 45:223-241

Matschinsky FM (2002) Regulation of pancreatic beta-cell glucokinase: from basics to therapeutics. Diabetes 51:S394-S404

Matschinsky FM, Glaser B, Magnuson MA (1998) Pancreatic betacell glucokinase: closing the gap between theoretical concepts and experimental realities. Diabetes 47:307-315

Merritt EA, Bacon DJ (1997) Raster3D: photorealistic molecular graphics. Methods Enzymol 277:505-524

Miller SP, Anand GR, Karschnia EJ, Bell GI, LaPorte DC, Lange AJ (1999) Characterization of glucokinase mutations associated with maturity-onset diabetes of the young type 2 (MODY-2): different glucokinase defects lead to a common phenotype. Diabetes 48:1645-1651

Njolstad PR, Cockburn BN, Bell GI, Sovik O (1998) A missense mutation, Val62Ala, in the glucokinase gene in a Norwegian family with maturity-onset diabetes of the young. Acta Paediatr 87:853-856

Njolstad PR, Sagen JV, Bjorkhaug L, Odili S, Shehadeh N, Bakry D, Sarici SU, Alpay F, Molnes J, Molven A et al (2003) Permanent neonatal diabetes caused by glucokinase deficiency: inborn error of the glucose-insulin signaling pathway. Diabetes 52:28542860 
Pedelini L, Garcia-Gimeno MA, Marina A, Gomez-Zumaquero JM, Rodriguez-Bada P, Lopez-Enriquez S, Soriguer FC, CuestaMunoz AL, Sanz P (2005) Structure-function analysis of the alpha5 and the alpha13 helices of human glucokinase: description of two novel activating mutations. Protein Sci 14:2080-2086

Stride A, Vaxillaire M, Tuomi T, Barbetti F, Njolstad PR, Hansen T, Costa A, Conget I, Pedersen O, Sovik O et al (2002) The genetic abnormality in the beta cell determines the response to an oral glucose load. Diabetologia 45:427-435

Velho G, Froguel P (1997) Maturity-onset diabetes of the young (MODY), MODY genes and non-insulin-dependent diabetes mellitus. Diabetes Metab 23:34-37
Velho G, Froguel P, Gloyn AL, Hattersley AT (2004) Maturity onset diabetes of the young type 2. In: Matschinsky FM, Magnuson MA (eds) Glucokinase and glycemic disease. From basics to novel therapeutics, Karger, Basel, pp 42-64

Zelent D, Najafi H, Odili S, Buettger C, Weik-Collins H, Li C, Doliba N, Grimsby J, Matschinsky FM (2005) Glucokinase and glucose homeostasis: proven concepts and new ideas. Biochem Soc Trans 33:306-310 